\title{
Higgs decay to fermions (CMS)
}

\author{
Pooja Saxena ${ }^{1, a}$ on behalf of the CMS colloboration \\ ${ }^{1}$ Deutsches Elektronen-Synchrotron, Notkestrasse 85, 22607 Hamburg, Germany
}

\begin{abstract}
A search for high mass Higgs boson of the Minimal Supersymmetric Standard Model decaying into two fermions using the first 2015 data at $13 \mathrm{TeV}$ is presented. The four final decay channels of $\mu \tau_{h}, e \tau_{h}, \tau_{h} \tau_{h}$ and $e \mu$ is used. The limits on production cross section times branching ratio has been set. Other results from Run1 and different searches and measurements involving Higgs decays fermions will also be reviewed.
\end{abstract}

\section{Introduction}

On 4th of July 2012, after the discovery of a particle with a mass near $125 \mathrm{GeV}$ by CMS and ATLAS collaboration, [1] [2] [3], many precise measurements of these properties have been performed: the signal strength, the latest mass measurement and the couplings with bosons ( $\gamma \gamma, \mathrm{ZZ}$ and WW) [4] [5]. All these measurements are compatible with a standard model (SM) Higgs boson [4] [5]. However, to claim that this newly observed particle is the SM Higgs boson, the couplings to the fermions has to be verified as well.

At the LHC, the Higgs boson can be produced in several ways, mainly by gluon-gluon fusion ( $\mathrm{ggH}$ ), also by Vector Boson Fusion (VBF), in association with a top quark pair (ttH), or in association with a $\mathrm{W} / \mathrm{Z}$ boson ( $\mathrm{VH})$. It mostly decays into a pair of b-quarks (57\%), in a pair of two $\tau$ leptons $(6.3 \%)$, and more rare into a pair of muons $(0.02 \%)$. In this document, results from Run-I on measurements involving Higgs decays to fermions ( $\tau \tau$, bb and combination of $\tau \tau$ and bb) will be reviewed in section 2 . Measurement of fermionic coupling is discussed in section 3. Further, measurements on universality of Higgs coupling flavour is address in section 4. Section 5 will report on the results on lepton flavour violation. A search for high mass Higgs boson of the MSSM decaying into two fermions using the first 2015 data at $13 \mathrm{TeV}$ is presented in section 6. Finally, conclusions are given in section 7.

\section{Higgs (125 GeV) Decay to fermions}

In this section, Higgs decay in fermionic decay mode ( $\tau \tau, b b$ and combination) will be presented. The results of bb measurement in vector boson fusion (VBF) mode will also be shown for first 2015 data at $13 \mathrm{TeV}$.

\footnotetext{
ae-mail: pooja.saxena@desy.de
} 


\subsection{Higgs boson decay to $\tau \tau$}

Among the Higgs decay to a di-lepton pair, the $\tau \tau$ is the one with the largest BR, because of the large event rate expected in the SM compared to the other leptonic decay modes. This section reports on the results of a search for a SM Higgs boson using final states with a pair of $\tau$ leptons in protonproton collisions at $\sqrt{s}=7$ and $\sqrt{s}=8 \mathrm{TeV}$ at the LHC. Entire dataset collected in 2011 and 2012 by the CMS experiment corresponding to an integrated luminosity of $4.9 \mathrm{fb}^{-1}$ at a centre of mass energy of $7 \mathrm{TeV}$ and $19.7 \mathrm{fb}^{-1}$ at $8 \mathrm{TeV}$ is being used [7]. All the possible decay modes of the di-tau system are used: $e \mu, e e, \mu \mu, e \tau_{h}\left(\tau_{h}\right.$ represent the hadronic decay mode of $\left.\tau\right), \mu \tau_{h}$ and $\tau_{h} \tau_{h}$. The main backgrounds are represented by $Z \rightarrow \tau \tau, Z \rightarrow l l, \mathrm{~W}+$ jets events and QCD processes. For most of the channels, the signal extraction is done with a likelihood fit on the di-tau mass. Events are categorized exploiting the number of jets, boost of the Higgs system and the sensitivity to ggH production according to the expected S/B. The "0-jet" categories are mostly used to constrain the main backgrounds. The combined observed and predicted $m_{\tau \tau}$ distributions for the $\mu \tau_{h}, e \tau_{h}, \tau_{h} \tau_{h}$, and $e \mu$ channels is shown in Fig 1 (left). An excess of events over the background-only hypothesis is observed with a local significance of 3.2 standard deviations at $m_{H}=125 \mathrm{GeV}$, while the expected significance is 3.7 standard deviations and the best-fit value for the signal strength, $\mu=\sigma / \sigma_{S M}$, is $0.78 \pm 0.27$ times the SM prediction, see Fig 1 (right), from which it can be conclude that there is clear evidence of the Higgs boson decays to pair of $\tau$ lepton.
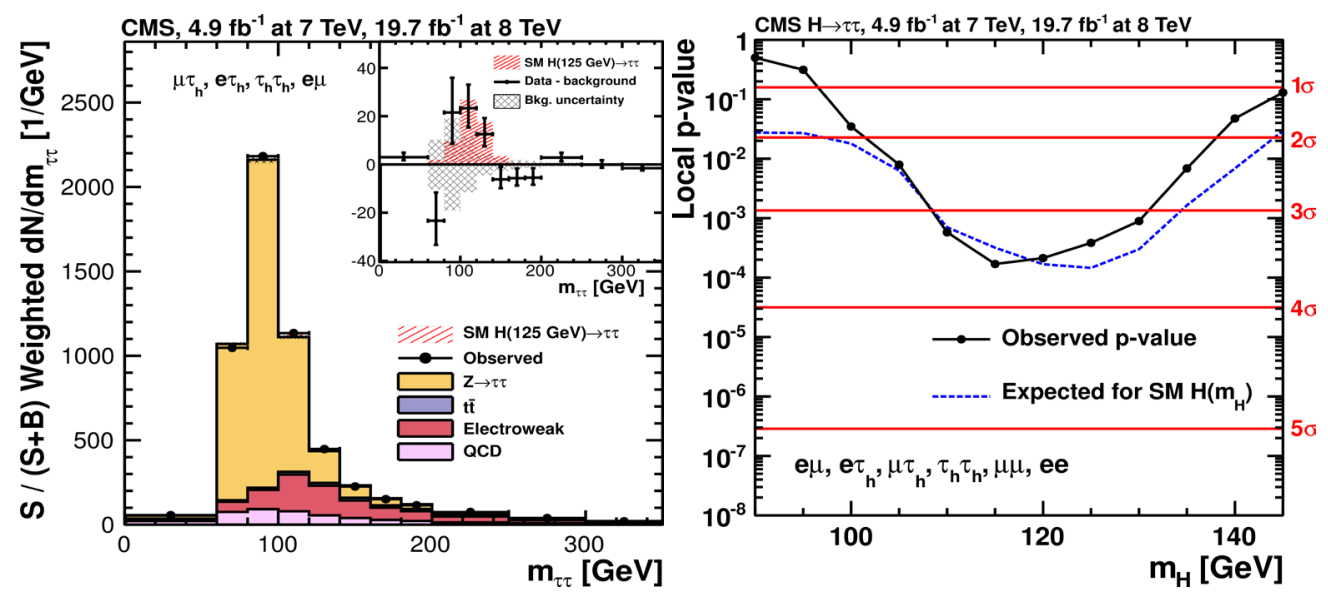

Figure 1. Combined observed and predicted $m_{\tau \tau}$ distributions for the $\mu \tau_{h}, e \tau_{h}, \tau_{h} \tau_{h}$, and $e \mu$ channels (left). Local p-value and significance in number of standard deviations as a function of the SM Higgs boson mass hypothesis (right)

\subsection{Higgs boson decay to bb}

At the LHC, in the mass range considered in this study, the vector boson fusion (VBF) process has the second largest production cross section following gluon fusion (GF) [8]. Furthermore, for a SM Higgs boson with a mass $m_{H}<135 \mathrm{GeV}$, the expected dominant decay mode is bb. Because of the huge QCD background, the ggH production is not considered. The signal topology of the production mechanism is exploited for $\mathrm{VH}$ and $\mathrm{VBF}$ production mode. 


\subsubsection{VH mode}

All the six channels for the leptonic decay of $\mathrm{V}$ are considered: $\mathrm{Z}(1 \mathrm{ll}) \mathrm{H}(\mathrm{bb})$ with $\mathrm{l}=\mathrm{e}, \mu, \mathrm{Z}(v v) \mathrm{H}(\mathrm{bb})$ and $\mathrm{W}(\mathrm{l} v) \mathrm{H}(\mathrm{bb})$ with $\mathrm{l}=\mathrm{e}, m u, \tau$. Data control regions are used to normalize the main contributions $(\mathrm{V}+\mathrm{jets}$, $t t$ and $\mathrm{VV}$ ) To increase the background rejection, the resolution on the b-jet pair mass (jets arising from the hadronization of a b-quark) is improve by $15-25 \%$ by applying a Multi Variate Analysis (MVA) regression. The event categorization is based on the high boost ( $100 \mathrm{GeV})$ of the $\mathrm{V}$ bosons, with bins in $\mathrm{pT}(\mathrm{V})$, and multivariate discriminators are used for signal extraction in 14 regions defined. An excess of events is observed with a local significance of 2.1 standard deviations at $\mathrm{mH}=125 \mathrm{GeV}$ (the expected significance is 2.1 standard deviations), and the best-fit value for the signal strength is $1.0 \pm 0.5$ times the SM prediction (see Fig. 2), and therefore shows a very good agreement with SM expectations.

Fig. 2 shows the combination of all channels into a single distribution. Events are sorted in bins of similar expected signal to background ratio, as given by the value of the output of their corresponding BDT discriminant (trained with a Higgs boson mass hypothesis of $125 \mathrm{GeV}$ ).

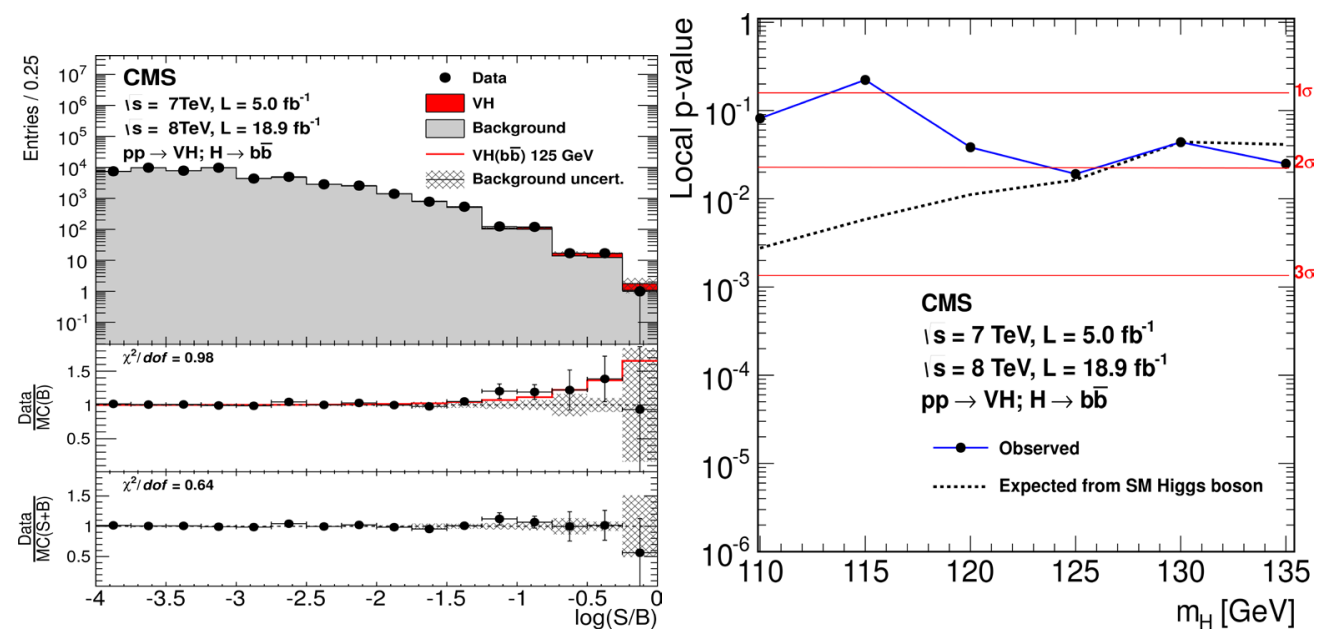

Figure 2. Combination of all channels into a single distribution (left). Local p values and corresponding significance (measured in standard deviations) for the background-only hypothesis to account for the observed excess of events in the data (right).

\subsubsection{VBF mode}

The data sample for this analysis corresponds to an integrated luminosity of $2.3 \mathrm{fb}^{-1}$ recorded in 2015 with the CMS experiment [9]. The prominent feature of the VBF process is the presence of four energetic jets in the final state. Further, the dominant background arise from QCD production of multijet events. Other backgrounds arise from hadronic decays of $\mathrm{Z}$ or $\mathrm{W}$ bosons produced in association with additional jets, hadronic decays of singly produced top quarks and hadronic decays of top quark pairs. The search is performed on selected four-jet events that are characterized by the response of a multivariate discriminant trained to separate signal events from background without making use of kinematic information on the two b-jet candidates. Distributions of the invariant mass of the two VBF-jet candidates for events in the DoubleB samples is shown in Fig. 3 (left). Observed 
and SM-expected likelihood profile of the signal strength $\mu=\sigma / \sigma_{S M}$ with $m_{H}=125 \mathrm{GeV}$, using 8 $\mathrm{TeV}$ data of Run-I and $13 \mathrm{TeV}$ data of Run 2 and for the combination of $8 \mathrm{TeV}$ and $13 \mathrm{TeV}$ data (right). The expected upper limits in the absence of a signal is 5.0 times the SM prediction, while the observed upper limit is 3.0, and the fitted signal strength is $\mu=\sigma / \sigma_{S M}=-3.7_{-2.5}^{+2.4}$. The combination of the results obtained in this search with the similar CMS search with Run 1 data yields an observed (expected) upper limit of 3.4 (2.3) times the SM prediction. The combined fitted signal strength is $\mu$ $=1.3_{-1.1}^{+1.2}$, with a signal significance of 1.2 standard deviations for $m_{H}=125 \mathrm{GeV}$, see Fig. 3 (right).
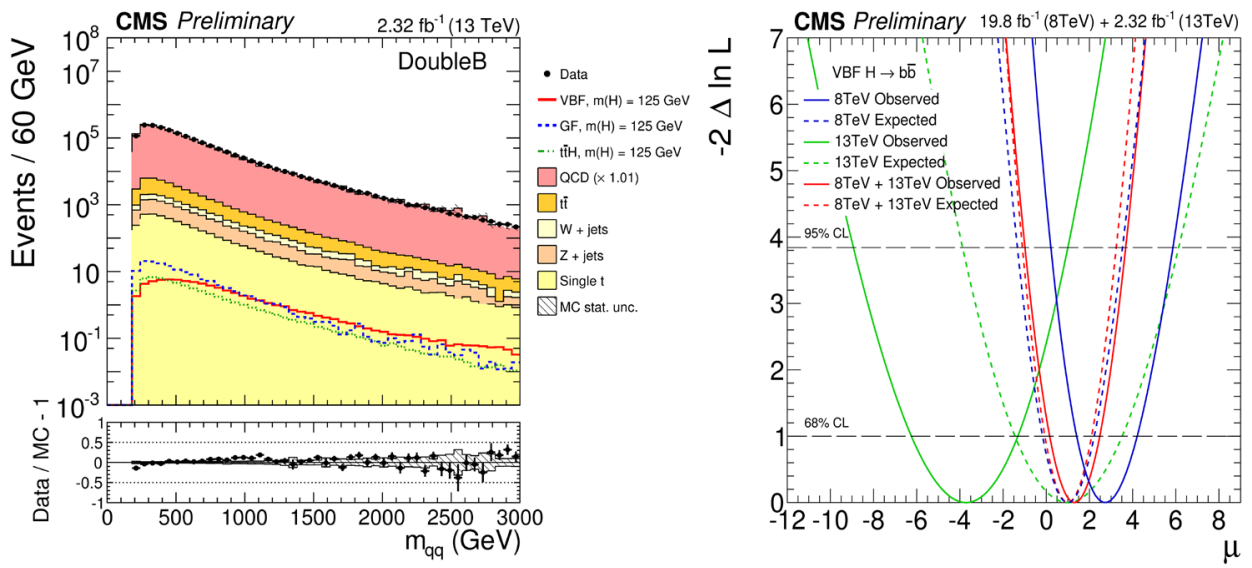

Figure 3. Distributions of the invariant mass of the two VBF-jet candidates (mqq) for events in the DoubleB samples (left). Observed and SM-expected likelihood profile of the signal strength $\mu=\sigma / \sigma_{S M}$ with $m_{H}=125$ $\mathrm{GeV}$, using $8 \mathrm{TeV}$ data of Run-I and $13 \mathrm{TeV}$ data of Run 2 and for the combination of $8 \mathrm{TeV}$ and $13 \mathrm{TeV}$ data (right).

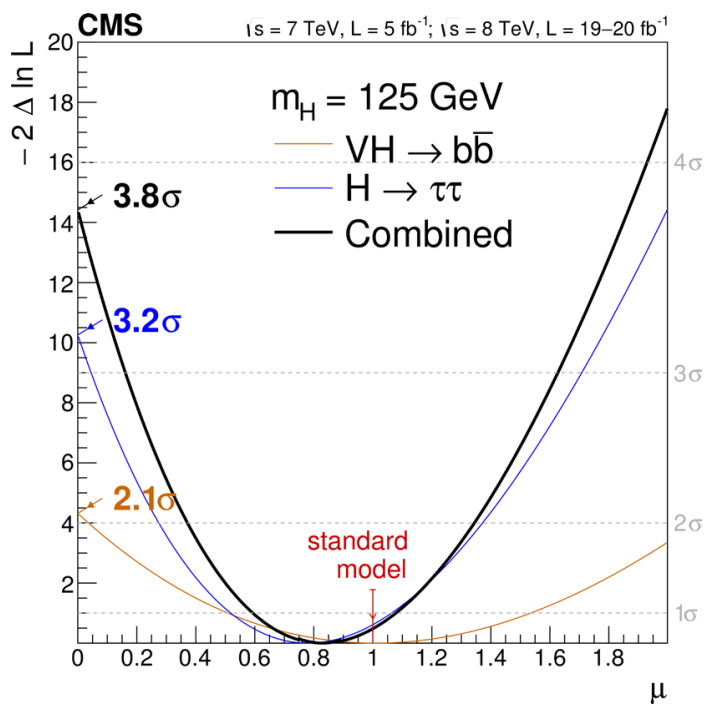

Figure 4. Scan of the profile likelihood as a function of the signal strength relative to the expectation for the production and decay of a standard model Higgs boson, $\mu$, for $m_{H}=125 \mathrm{GeV}$. 


\subsection{Fermionic combination}

A combination of the Higgs boson decays to $\tau \tau$ channel and Higgs boson decays to bb (VH only) is performed [10]. This combination results in strong evidence for the direct coupling of the $125 \mathrm{GeV}$ Higgs boson to fermions, with an observed (expected) significance of 3.8 (4.4) standard deviations (see Fig. 4).

\section{Measurement of fermionic coupling}

Fig 5 shows fits for deviations in the coupling for a generic five-parameter model [14] assuming effective loop couplings according to the SM. For the fermions, the values of the fitted Yukawa couplings are shown, while for vector bosons the square-root of the coupling for the hVV vertex divided by twice the vacuum expectation value of the Higgs boson field is shown. The linear dependence on the mass parameter nicely illustrates the validity of the Higgs mechanism providing the masses of the fundamental particles in the SM.

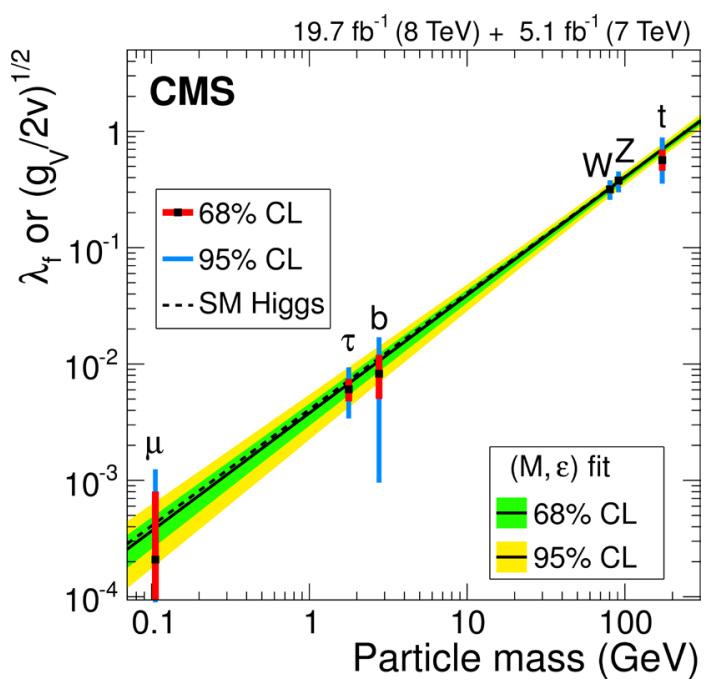

Figure 5. Summary of the fits for deviations in the coupling using the SM loop induced couplings, expressed as function of the particle mass.

\section{Universality of Higgs coupling flavour}

In this section, measurement of Higgs boson coupling to first and second generation leptons will be presented [12]. The analysis is performed by directly combining the full $\sqrt{s}=7 \mathrm{TeV}$ and $\sqrt{s}=8 \mathrm{TeV}$ datasets $\left(5.0+19.7 \mathrm{fb}^{-1}\right)$, using the ggH and VBF production modes (for the $\mathrm{VH}$ and ttH production mode the branching ratio is too small). The SM predicts the coupling of the new boson to leptons is flavour-universal or proportional to the lepton mass. However, the branching ratio for $H \rightarrow \mu \mu$ is very low $\left(2.2 \times 10^{-4}\right)$, and even smaller for $H \rightarrow e e$. One of the important feature of this analysis is the clean signature in the detector.

Events are categorized based on the number or reconstructed jets attempting to separate the gluongluon fusion and VBF production components. The signal is extracted by means of a fit to the $m_{\mu \mu}$ distribution using signal and background shapes. As no significant $H \rightarrow \mu \mu$ is observed, upper limits on the cross section times the $H \rightarrow \mu \mu$ branching ratio are set. The combined observed (expected) limit on the signal strength, for a Higgs boson with a mass of $125 \mathrm{GeV}$, is $7.4\left(6.5_{-1.9}^{+2.8}\right)$, Fig. 6 (left). 
Assuming the SM production cross section, this corresponds to an upper limit of 0.0016 on $\beta(H \rightarrow$ $\mu \mu$ ). For $H \rightarrow e e$, SM Higgs boson decays are far too rare to detect, and no signal is observed. For a Higgs boson mass of $125 \mathrm{GeV}$, a $95 \% \mathrm{CL}$ upper limit of $0.041 \mathrm{pb}$ is set on $\sigma \beta(H \rightarrow e e)$ at $8 \mathrm{TeV}$, see Fig. 6 (right).

These results, together with the above quoted evidence for the $125 \mathrm{GeV}$ boson coupling to $\tau$ leptons confirm the SM prediction that the leptonic couplings of the new boson are not flavour universal.
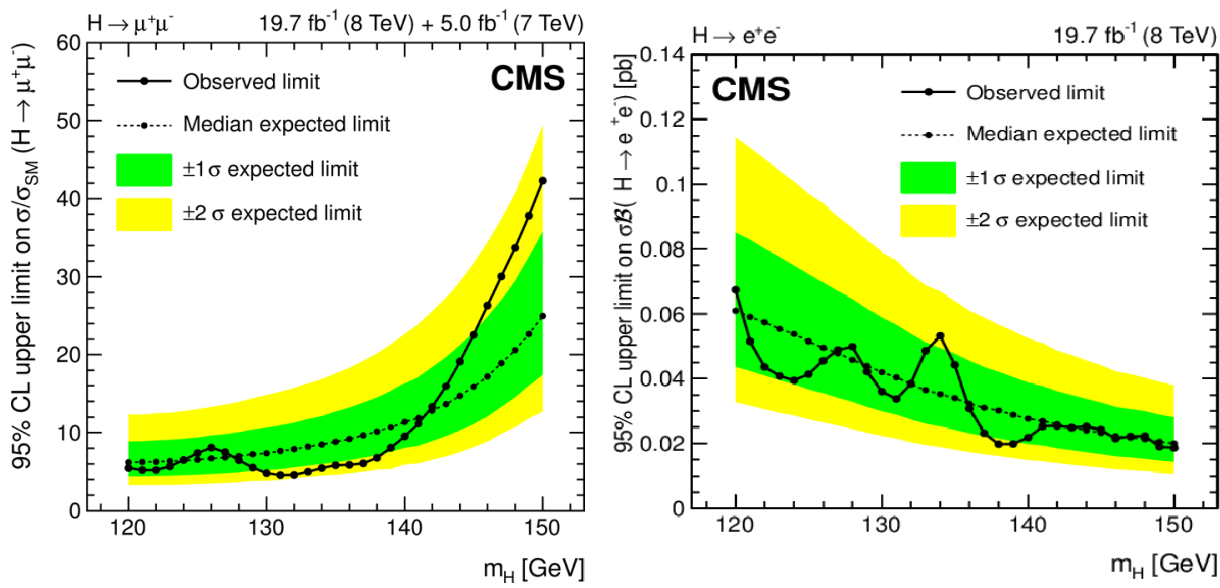

Figure 6. Mass scan for the background-only expected and observed combined exclusion limits for $H \rightarrow \mu \mu$ (left). Exclusion limits on $\sigma \beta$ shown for $H \rightarrow e e$ (right).

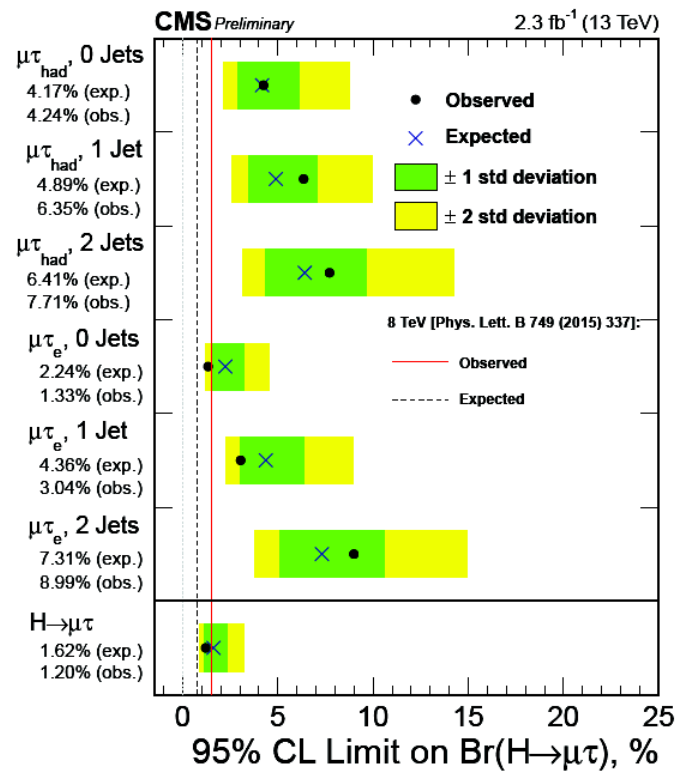

Figure 7. Observed and expected $95 \%$ CL upper limits on the $B(H \rightarrow \mu \tau)$ for each individual category and combined. 


\section{Lepton flavour violation (LFV)}

In this section, a direct search for lepton flavour violating decays of the Higgs boson in the $H \rightarrow \mu \tau$ channel using $2.3 \mathrm{fb}^{-1}$ data at $\sqrt{s}=13 \mathrm{TeV}$ with the CMS experiment [11]. In the SM, LFV decays of the Higgs boson are forbidden, which make it an interesting observation. The search is performed in two channels, $H \rightarrow \mu \tau_{e}$ and $H \rightarrow \mu \tau_{h}$, where $\tau_{e}$ and $\tau_{h}$ are tau leptons reconstructed in the electronic and hadronic decay channels, respectively. This analysis follow the procedure of the $\sqrt{s}=$ $8 \mathrm{TeV}$ analysis, where a small excess was observed, corresponding to $2.4 \sigma$, with an analysis based on an integrated luminosity of $19.7 \mathrm{fb}^{-1}$ that yielded an expected 95\% CL limit on the branching fraction of $0.75 \%$. No excess is observed in $\sqrt{s}=13 \mathrm{TeV}$ analysis. The best-fit branching fraction is $B(H \rightarrow \mu \tau)=-0.76_{-0.84}^{+0.81} \%$ and an upper limit of $B(H \rightarrow \mu \tau)<1.20 \%$ (1.62\% expected) is set at $95 \%$ CL, see Fig. 7
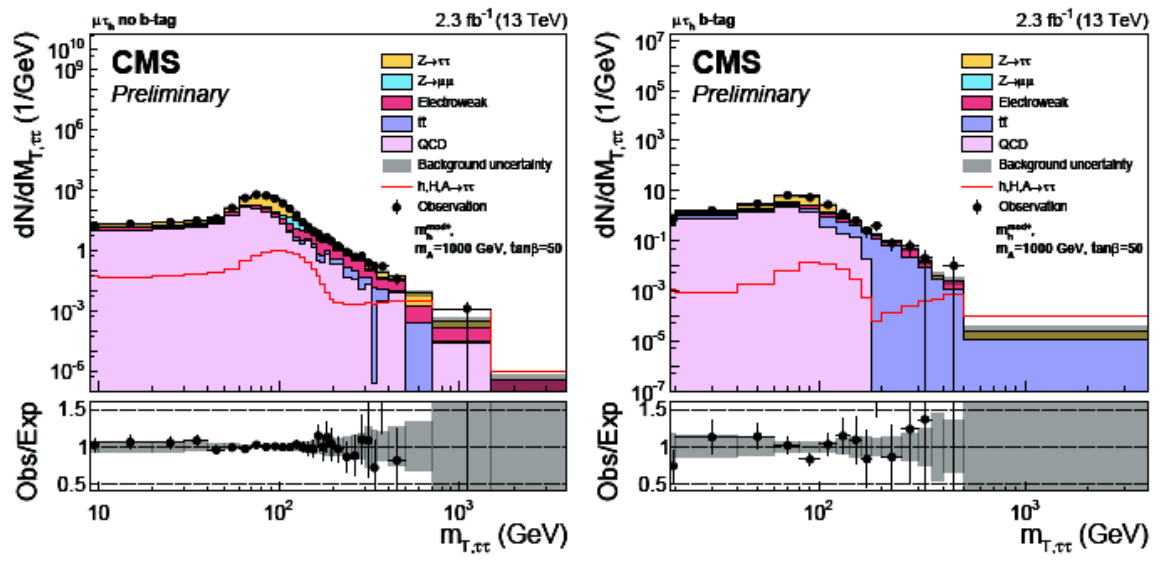

Figure 8. Post-fit plot of the transverse mass distribution in the no b-tag category (left) and the b-tag category of the $e \tau_{h}$ channel (right).

\section{Minimal Supersymmetric Standard Model (MSSM)}

This search is performed on a dataset corresponding to an integrated luminosity of $2.3 \mathrm{fb}^{-1}$ of pp collision data at a centre-of-mass energy of $13 \mathrm{TeV}$, collected by CMS in 2015 [13]. As number of arguments favour the existence of theories beyond the SM, many versions of which include an extended Higgs sector. One of the simplest extensions of the SM is the MSSM.

Four final states of the tau pair are considered: $e \mu, e \tau_{h}, \mu \tau_{h}$ and $\tau \tau$. The methedology of this analysis is to stay close to analysis performed at $8 \mathrm{TeV}$, though the physics objects, selection and analysis tools are re-optimized. The dominant production mode is gluon fusion (ggf) and b-associated production (bbf).

A binned maximum likelihood fit is performed simultaneously to the transverse mass distributions in the b-tag and no b-tag categories and all four channels Fig. 8. As no significant excess of data over the background predictions is found, we set 95\% CL upper limits on cross-section times branching fraction for the two dominant production modes, ggf and bbf. Fig. 9 shows these limits as a function of $m_{\phi}$. Finally, Fig. 10 shows the model dependent limits for two different benchmark models, the $m_{h}^{\text {mod }+}$ and hMSSM scenarios. 

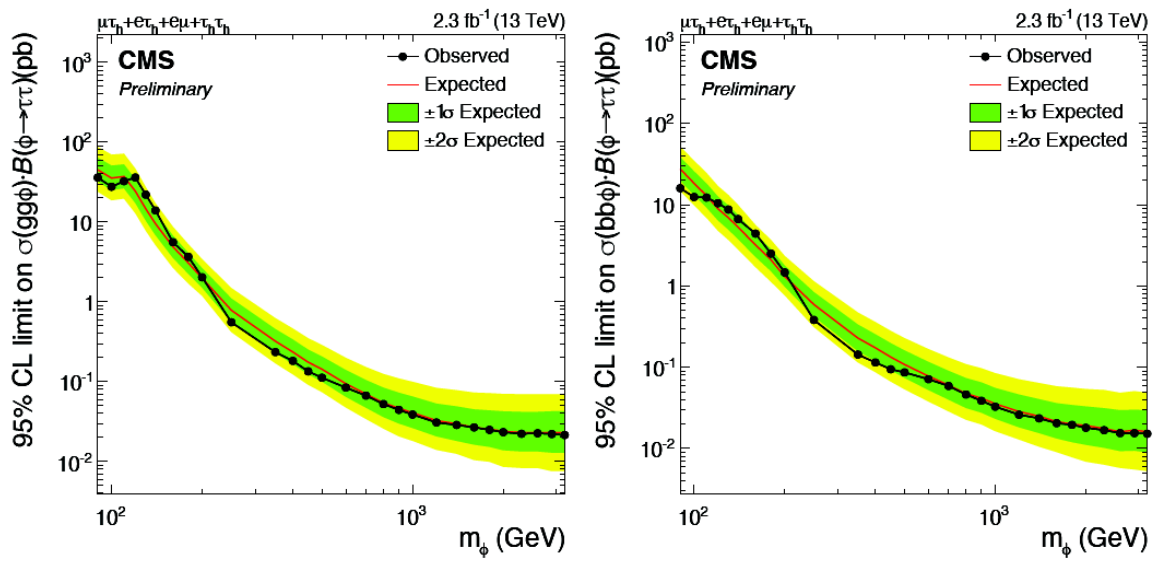

Figure 9. Expected and observed limits on cross-section times branching fraction for the gluon fusion process (left) and the b-associated production process (right), resulting from the combination of all four channels.
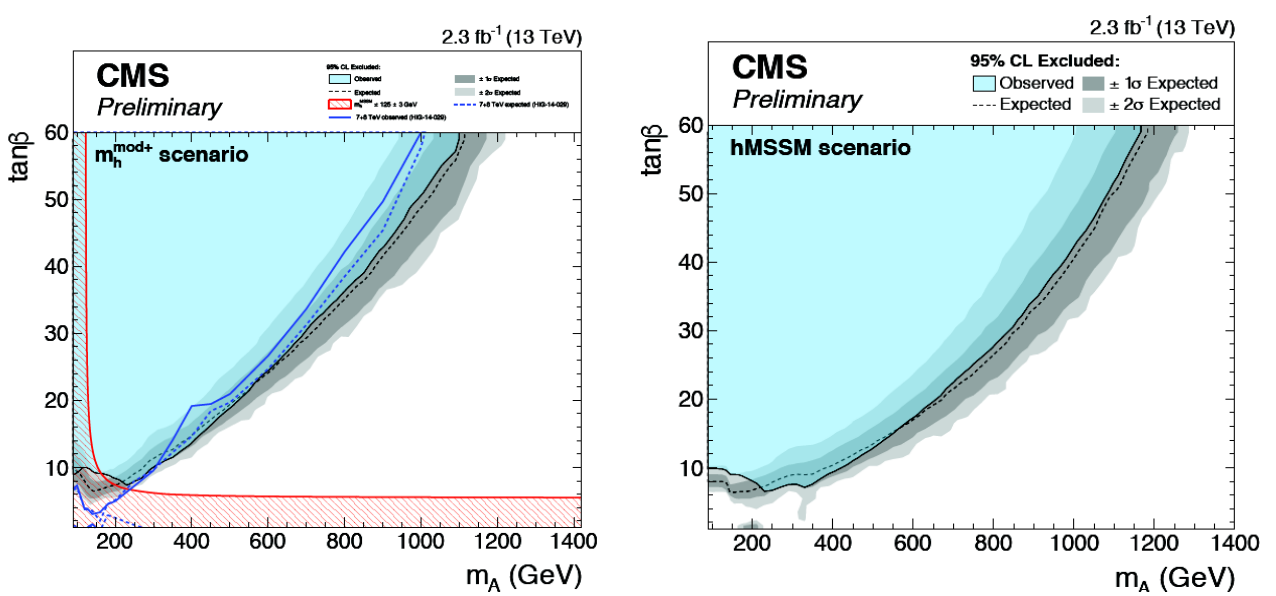

Figure 10. Model dependent exclusion limits in the $m_{A}-\tan \beta$ plane, combining all channels, for the $m_{h}^{\text {mod }+}$ (left) and hMSSM (right) scenarios.

\section{Summary}

Searches for a SM Higgs boson decaying to fermions have been performed in CMS for different channels, $H \rightarrow \tau \tau, H \rightarrow b b$ and $H \rightarrow \mu \mu$, using different production modes, and there is a strong evidence of the Higgs decays to fermions. The excess seen for $H \rightarrow \tau \tau$ is of $3.2 \sigma$ and constitutes by itself an evidence, the excess in the $H \rightarrow b b$ (VH channel) is of $2.1 \sigma$, and combining these two channels, it leads to an evidence of $3.8 \sigma$ for the Higgs decaying to fermions. 


\section{References}

[1] CMS Collaboration, Phys. Lett. B716 30 (2012).

[2] CMS Collaboration, JHEP06 081 (2013) .

[3] ATLAS Collaboration, Phys. Lett. B716 1 (2012).

[4] CMS Collaboration, JHEP01 096 (2014).

[5] CMS Collaboration, Phys. Rev. D89 092007 (2014).

[6] CMS Collaboration, JINST3 S08004 (2008)

[7] CMS collaboration, J. High Energ. Phys. 104 (2014), doi:10.1007/JHEP05(2014)104

[8] LHC Higgs Cross Section Working Group, CERN Report CERN-2012-002, 2012. 534 doi:10.5170/CERN-2012-002, arXiv:1201.3084.

[9] CMS Collaboration, CMS-PAS-HIG-16-003.

[10] CMS collaboration, Nature Physics 10, 557-560 (2014) doi:10.1038/nphys3005

[11] CMS Collaboration, CMS-PAS-HIG-16-005.

[12] CMS Collaboration, Phys. Lett. B744 (2015) 184-207

[13] CMS Collaboration, CMS-PAS-HIG-16-006. 